\title{
Impacto da campanha "Considere o Risco", da Sociedade Brasileira de Ortopedia e Traumatologia, na percepção do risco e na utilização do checklist cirúrgico por ortopedistas brasileiros*
}

\section{Impact of the "Mind the risk" Campaign of Sociedade Brasileira de Ortopedia e Traumatologia on Risk Perception and Use of the Surgical Checklist by Brazilian Orthopedists}

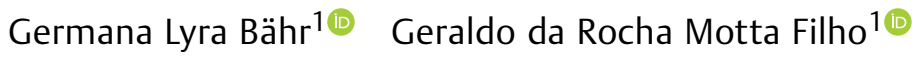 \\ ${ }^{1}$ Divisão de Traumatologia e Ortopedia, Instituto Nacional de \\ Traumatologia e Ortopedia (Into), Rio de Janeiro, RJ, Brasil \\ Rev Bras Ortop 2021;56(2):218-223. \\ Endereço para correspondência Geraldo da Rocha Motta Filho, MD, \\ MSc, Instituto Nacional de Traumatologia e Ortopedia (Into), Rua \\ Raimundo de Magalhães, 92, Gávea, Rio de Janeiro, RJ, 22451-150, \\ Brasil (e-mail: geraldomotta@terra.com.br).
}

\section{Resumo}

Palavras-chave

- segurança do paciente

- erros médicos

- procedimentos cirúrgicos

- lista de verificação
Objetivo Analisar o impacto das ações educacionais inseridas na campanha "Considere o Risco", da Sociedade Brasileira de Traumatologia e Ortopedia (SBOT), para aumentar a percepção do risco envolvido na atividade cirúrgica e a utilização do checklist cirúrgico.

Métodos Realização de pesquisa comparativa, durante o $50^{\circ}$ Congresso Brasileiro de Ortopedia e Traumatologia ( $50^{\circ} \mathrm{CBOT}$ ), em novembro de 2018 , utilizando questionário semelhante ao de duas versões anteriores.

Resultados O número de participantes foi de 730 , correspondendo à $18,7 \%$ do total de 3903 inscritos no $50^{\circ}$ CBOT. No total, 542 ortopedistas $(74,2 \%)$ relataram já ter vivenciado erros dentro do centro cirúrgico e $218(29,8 \%)$ cirurgias em locais errados; 624 participantes $(85,5 \%)$ afirmaram marcar o local da cirurgia e 402 (55\%) utilizar regularmente o checklist cirúrgico.

Conclusão Na amostra pesquisada, ficou evidenciado que os esforços da SBOT para a disseminação do conhecimento do protocolo da Organização Mundial de Saúde (OMS) foram efetivos, reduzindo a quantidade de ortopedistas que o desconheciam de 65,3\% (em 2012) para 20,7\% (em 2018), e ampliando sua utilização. Em 2018, 402 ortopedistas (55\% da amostra) referiram fazer uso frequente do protocolo no ambiente cirúrgico, em

Trabalho desenvolvido no Instituto Nacional de Traumatologia e Ortopedia (Into), Rio de Janeiro, RJ, Brasil.

recebido

24 de Junho de 2019

aceito

30 de Outubro de 2019

Publicado online

Abril 2, 2020
DOI https://doi.org/ $10.1055 / \mathrm{s}-0040-1701285$ ISSN 0102-3616. (c) 2020. Sociedade Brasileira de Ortopedia e Traumatologia. All rights reserved.

This is an open access article published by Thieme under the terms of the Creative Commons Attribution-NonDerivative-NonCommercial-License, permitting copying and reproduction so long as the original work is given appropriate credit. Contents may not be used for commercial purposes, or adapted, remixed, transformed or built upon. (https://creativecommons.org/ licenses/by-nc-nd/4.0/)

Thieme Revinter Publicações Ltda., Rua do Matoso 170, Rio de Janeiro, RJ, CEP 20270-135, Brazil 


\section{Abstract}

\section{Keywords}

- patient safety

- medical errors

- surgical procedures

- checklist comparação com 301 (40,2\%) em 2014. Estes dados confirmam a necessidade de campanhas educacionais e treinamentos sistemáticos, não apenas para promover uma mudança de comportamento, como também, principalmente, uma mudança cultural.

Objective To analyze the impact of the educational actions included in the "Mind the Risk" campaign of Sociedade Brasileira de Traumatologia e Ortopedia (Brazilian Society of Traumatology and Orthopedics, SBOT, in Portuguese), to increase the perception of the risk involved in the surgical activity and the use of the surgical checklist.

Methods A comparative research was performed during the 50th Brazilian Congress on Orthopedics and Traumatology (50 CBOT, in Portuguese) in November 2018, using a questionnaire similar to the one used in previous two versions.

Results The number of participants was 730 , corresponding to $18,7 \%$ of the total of 3903 enrolled in the $50^{\circ} \mathrm{CBOT}$. Among the participants, $542(74,2 \%)$ reported having experienced errors within the surgical units and $218(29,8 \%)$ surgeries in wrong sites. In total, $624(85,5 \%)$ reported marking the surgical site and 402 (55\%) reported using the surgical checklist systematically.

Conclusion In the sample studied, it was evidenced that SBOT's efforts to disseminate the World Health Organization (WHO) protocol were effective, reducing the number of orthopedists who were unaware of it from 65.3\% (in 2012) to 20.7\% (in 2018), and expanding its use. In 2018, 402 participants (55\%) reported the systematic use of the protocol compared with $301(40,2 \%)$ in 2014. These data confirm the need for educational campaigns and systematic training, not only to promote behavioral change, but especially a cultural change.

\section{Introdução}

A preocupação com o risco envolvido no exercício da Medicina remonta à Antiguidade, conforme demonstra o princípio primum non nocere (em primeiro lugar, não causar danos), atribuído a Hipócrates.

Sociedades médicas no mundo reconheceram e lideraram o movimento para coibir os erros médicos e o estabelecimento dos conceitos de cirurgia segura. A American Academy of Orthopaedic Surgeons - (Academia Americana de Cirurgiões Ortopédicos, AAOS, em inglês) iniciou seus esforços com a iniciativa conhecida como "Wrong-Site Surgery" ainda nos anos 1980, publicando seus resultados preliminares em $1984 .^{1-3}$

Em 2000, a publicação do Institute of Medicine (Instituto de Medicina, IOM, em inglês), intitulada To Err is Human: Building a Safer Health System, ${ }^{4}$ sensibilizou o público, a mídia, os políticos e os profissionais médicos, e consolidou o interesse pelo tema.

Em 2002, os países membros da Organização Mundial de Saúde (OMS), após reconhecerem a necessidade de reduzir o dano e o sofrimento de pacientes e familiares advindo de erros médicos, acordaram em uma resolução para aumentar a segurança do paciente dentro das políticas públicas mundiais e, em 2004, a OMS criou a Aliança Mundial para a Segurança do Paciente, que, a partir de 2005, passou a definir temas prioritários a serem abordados a cada 2 anos, conhecidos como "Desafios Globais." 5

Entre 2007 e 2008, o segundo desafio global (Cirurgia Segura) estabeleceu como objetivo a melhoria da segurança no ambiente cirúrgico, com o objetivo de aumentar os padrões de qualidade e segurança do cuidado cirúrgico por meio de 4 ações importantes: 1) prevenção de infecções do sítio cirúrgico; 2) anestesia segura; 3 ) equipes cirúrgicas seguras; e 4) indicadores da assistência cirúrgica. ${ }^{5}$ Com base nestas ações, foi iniciada, nos países membros da OMS, uma campanha conhecida como "Cirurgias Seguras Salvam Vidas".

Em 2008, o Ministério da Saúde do Brasil aderiu à campanha, cujo principal objetivo era a adoção, pelos hospitais, de uma lista de verificação padronizada contendo 19 itens, elaborada para melhorar a comunicação entre os membros da equipe cirúrgica, reduzir os riscos de erros e danos ao paciente, e diminuir complicações e mortes associadas com as cirurgias, conhecida como checklist cirúrgico, a ser realizado em todas as cirurgias e em três fases: antes do início da anestesia (Sign in), antes da incisão na pele (Time Out), e antes da saída do paciente da sala cirúrgica (Sign out) (- Figura 1). ${ }^{6}$

A utilização do checklist cirúrgico proposto pela OMS é obrigatória nos Estados Unidos, Canadá, Inglaterra e Jordânia, mas, em muitos países, como no Brasil, é apenas sugerida.

Dois importantes estudos retrospectivos ${ }^{7,8}$ sugerem que pelo menos $50 \%$ dos eventos adversos cirúrgicos são evitáveis. A maioria destes eventos não é causada por problemas técnicos, mas pela falta de trabalho em equipe, liderança, comunicação, tomada de decisão e consciência situacional, pois mesmo os procedimentos mais simples envolvem dezenas de etapas críticas, com inúmeras oportunidades para falhas e enorme potencial de erros, resultando em lesões aos pacientes. ${ }^{9,10}$

O obstáculo mais crítico para aumentar a segurança no ambiente cirúrgico é a falta de percepção do risco por boa parte dos profissionais envolvidos, principalmente os 


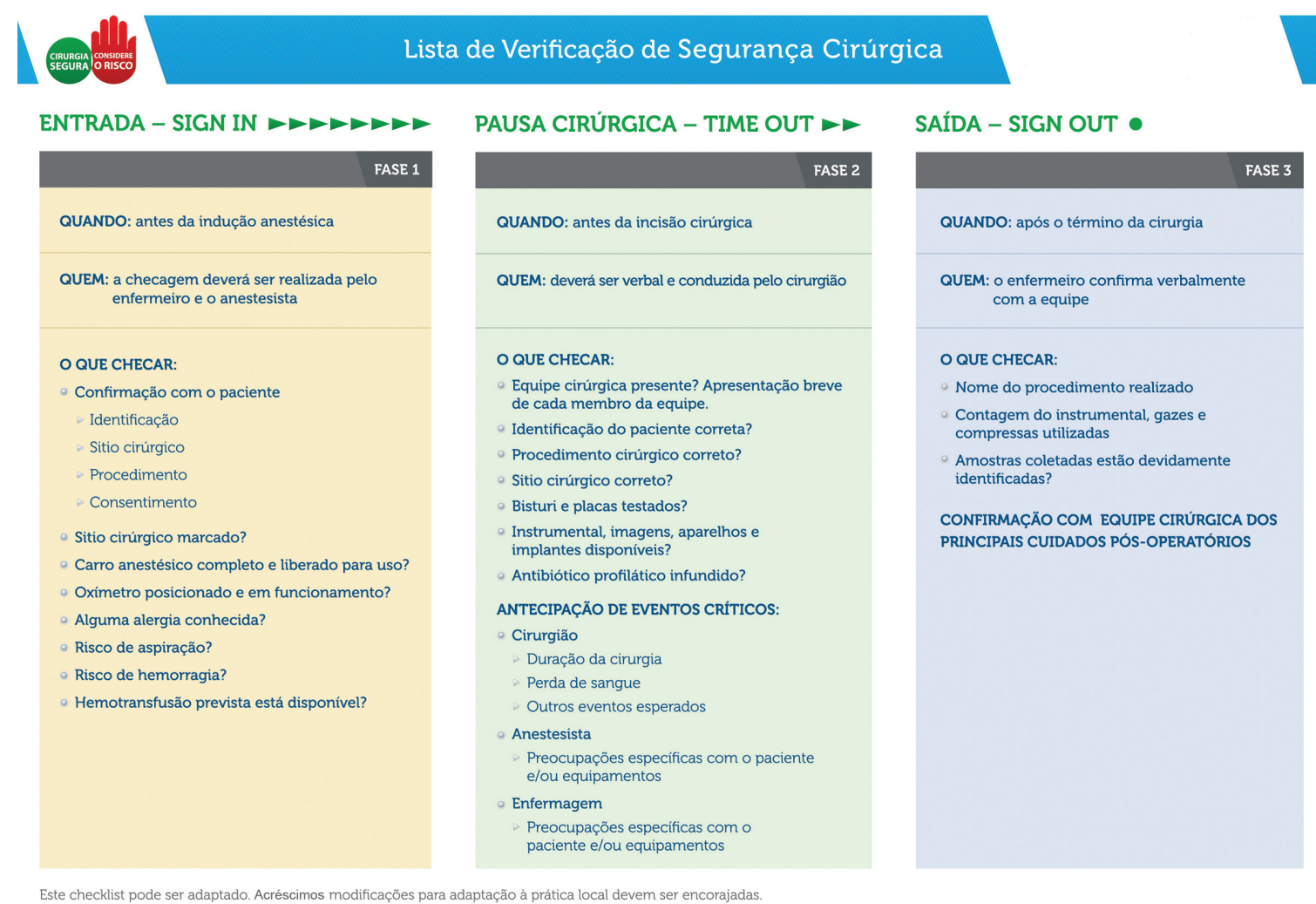

Fig. 1 Lista de verificação (checklist cirúrgico) proposta pela Organização Mundial de Saúde (OMS) e adaptada para uso em nossa instituição.

cirurgiões. Além disso, falhas na comunicação entre os membros de uma equipe podem interferir no seu desempenho e na segurança do paciente. Uma equipe que trabalhe unida para usar seus conhecimentos e habilidades em benefício do paciente, combinando precisão técnica com segurança, pode prevenir uma proporção considerável das complicações que ameaçam a vida. 9,10

Neste contexto, o uso correto de ferramentas como o Protocolo de Cirurgia Segura da OMS pode ajudar a atingir esta meta, inclusive melhorando a comunicação entre os membros da equipe envolvidos na cirurgia. ${ }^{11}$

Segundo um estudo ${ }^{12}$ publicado no Journal of Bone and Joint Surgery Reviews em 2016, a real incidência de cirurgias em locais errados em ortopedia é desconhecida, devido à falta dos números exatos de procedimentos realizados e de infraestrutura para uniformizar as notificações de erros. O estudo revela, porém, que $21 \%$ dos cirurgiões de mão, $50 \%$ dos cirurgiões de coluna, e 8,3\% dos cirurgiões de joelho relataram já ter vivenciado ao menos uma cirurgia em local errado durante sua carreira. O estudo conclui que todo cirurgião ortopédico encontra-se em risco de realizar uma cirurgia em local errado durante sua prática, e que a prevenção deveria ser uma prioridade na ortopedia. Além disso, sugere que, além de fatores como a liderança dos cirurgiões, o compromisso e a vigilância contínua, processos que estimulam a comunicação efetiva da equipe, o uso de checklists, e a coleta e a análise de dados devem ser utilizados em ambientes cirúrgicos ortopédicos, para a melhoria da segurança do paciente. ${ }^{12}$

Visando diagnosticar a percepção da segurança em ambiente cirúrgico e o grau de utilização do Protocolo de Cirurgia Segura da OMS por cirurgiões ortopedistas no Brasil, foi realizada uma pesquisa em novembro de 2012, durante o $44^{\circ}$ Congresso Brasileiro de Ortopedia e Traumatologia ( $44^{\circ}$ CBOT). A pesquisa demonstrou que 328 dos 502 respondentes $(65,3 \%)$ desconheciam parcial ou totalmente o protocolo e que $70,5 \%$ (123) dos que o conheciam afirmavam não terem sido treinados para a sua utilização. ${ }^{13}$

Baseada nestes resultados, a Sociedade Brasileira de Ortopedia e Traumatologia (SBOT) lançou, em 2012, a campanha educacional "Considere o Risco," com o objetivo não apenas de aumentar a percepção dos cirurgiões ortopedistas acerca dos riscos envolvidos no ambiente cirúrgico, como também de disseminar o uso do checklist cirúrgico da OMS como barreira de prevenção contra erros. A campanha englobou várias ações educacionais para a disseminação de informações sobre segurança no ambiente cirúrgico, incluindo palestras em congressos, fóruns de discussão, e materiais informativos, disponíveis no site da SBOT e veiculados em revistas da especialidade, faixas e folhetos.

O objetivo deste trabalho foi analisar o impacto das ações educacionais inseridas na campanha "Considere o Risco" da SBOT, para aumentar a percepção do risco envolvido na atividade cirúrgica e incentivar a utilização do checklist cirúrgico como barreira de proteção contra erros, após seis anos do seu lançamento.

\section{Materiais e Métodos}

O presente estudo é uma pesquisa de natureza exploratória, de caráter quantitativo, realizada mediante aplicação de um questionário sobre o tema "Cirurgia Segura" a 3.903 ortopedistas que participaram do $50^{\circ} \mathrm{CBOT}$, organizado pela SBOT no Rio de 
Janeiro, em novembro de 2018. O questionário, composto de questões semelhantes às utilizadas nos questionários aplicados aos ortopedistas brasileiros em 2012 , por ocasião do $44^{\circ} \mathrm{CBOT}$, e em 2014, por ocasião do $46^{\circ} \mathrm{CBOT}$, foi baseado no realizado pela AAOS, que, por sua vez, utilizou o questionário desenvolvido pela American Academy of Otholaryngology - Head and Neck Surgery (Academia Americana de Otolaringologia -Cirurgia de Pescoço e Cabeça, AAO-HNS, em inglês), modificado para se adequar à prática da ortopedia e traumatologia. ${ }^{14,15}$

O projeto de pesquisa foi aprovado pelo Comitê de Ética em Pesquisa do Instituto Nacional de Traumatologia e Ortopedia (Into) com o número CAAE (Sisnep) 36204914.0.0000.5273.

Foi utilizada a ferramenta de coleta e análise de dados SurveyMonkey (SurveyMonkey, San Mateo, CA, EUA), que permitiu a avaliação eficiente e rápida dos resultados. A distribuição dos questionários foi realizada por e-mail para todos os participantes do congresso, em três momentos diferentes, com intervalo de dez dias, caso não houvesse resposta depois do primeiro envio. 0 prazo de resposta foi de um mês após o primeiro envio.

O grupo de profissionais que respondeu à pesquisa não foi selecionado por nenhum critério específico que não a vontade de participar do estudo. Dessa forma, o tamanho da amostra foi aleatório.

\section{Resultados}

O número de profissionais participantes no $50^{\circ} \mathrm{CBOT}$ foi de 3.903, enquanto o número de formulários retornados foi de 730 , representando $18,7 \%$ do total.

A maior parte dos respondentes, 237 ortopedistas (32,5\%) tem sua área de atuação na ortopedia geral. Dentre os que atuam em subespecialidades, a cirurgia do joelho foi a que apresentou maior quantidade, com 133 ortopedistas (18,2\%), seguida pelo trauma ortopédico com $65(8,9 \%)$ e pela cirurgia do quadril representada por 59 profissionais $(8,1 \%)$.

Em relação à área geográfica, os ortopedistas participantes da pesquisa atuam em todos os estados do Brasil, com exceção de Sergipe, Rondônia, Amapá e Roraima. O estado de São Paulo foi o de maior representatividade na amostra, com um total de 199 respondentes (27,3\%), seguido do Rio de Janeiro com 197 (27\%) e Minas Gerais com 73 (10\%).

Entre estes 730 ortopedistas, 572 (78,4\%) afirmaram ter concluído residência médica em ortopedia e traumatologia.

A análise do tempo de exercício profissional deste grupo demonstrou que 278 (38,1\% do total), tem menos de 5 anos de atuação, 89(12,2\%) tem entre 5 e 10 anos, 151 (20,7\%) entre 10 e 20 anos e 212 (29\%) tem mais de 20 anos de prática.

Dentre os respondentes, 551 (75,5\%) afirmaram possuir título de especialista pela SBOT.

No total, 423 dos ortopedistas que responderam (58\%) espontaneamente à pesquisa não estavam envolvidos com as atividades científicas do congresso.

Ao todo, 624 ortopedistas da amostra (85,5\%) informaram marcar o local a ser operado antes de encaminhar o paciente ao centro cirúrgico, enquanto quase o mesmo percentual, 599 (82\%), relatou conferir o material de implante e o funcionamento dos equipamentos da sala antes da anestesia.
Tabela 1 Profissionais que não marcam o sítio cirúrgico

\begin{tabular}{|l|l|l|l|}
\hline & 2012 & 2014 & 2018 \\
\hline $\begin{array}{l}\mathrm{N}^{\circ} \text { total de } \\
\text { respondentes }\end{array}$ & 502 & 748 & 730 \\
\hline $\begin{array}{l}\text { Não marcam o } \\
\text { sítio cirúrgico }\end{array}$ & $183(36,5 \%)$ & $269(36 \%)$ & $106(14,5 \%)$ \\
\hline
\end{tabular}

A categoria de erro mais frequente foi relacionada à material para uso cirúrgico incompleto ou danificado, identificado após o inrício do procedimento, correspondendo a 418 casos (72,3\% do total), seguida por problemas nos equipamentos ou instrumentos da sala cirúrgica com 395 casos (68,3\%). Destes incidentes, $413(71,4 \%)$ foram notificados, para que melhorias pudessem ser implementadas.

A vivência de algum erro dentro do centro cirúrgico durante sua prática foi relatada por 542 (74,2\%) dos profissionais respondentes ; 218 (29,8\%) afirmaram já ter vivenciado cirurgias em locais errados, e 36 (4,9\%), cirurgias em pacientes errados.

Apesar do reconhecimento do risco envolvido na realização de cirurgias, e do protocolo da OMS como uma barreira de segurança para o paciente, médico e instituição, por 505 $(87,2 \%)$ dos 579 respondentes, que relataram conhecer o protocolo em 2018, 151 (20,7\%) ortopedistas afirmaram ainda desconhecer total ou parcialmente este protocolo, e 365 (50\%) mencionaram não ter tido treinamento para a sua utilização. A utilização dessa ferramenta com regularidade foi relatada por 402 (55\%) ortopedistas.

Seis anos depois de iniciada a campanha da SBOT, foi possível identificar por meio do presente estudo que: 1. $14,5 \%$ dos cirurgiões (108) ainda não marcam o sítio cirúrgico em 2018, contra 36,5\% (183) em 2012 (-Tabela 1); 2. 20,7\% (151) ainda desconhecem o Protocolo de Cirurgia Segura da OMS, contra 65,3\% (328) em 2012 (-Tabela 2); 3. 69,1\% (505) reconhecem o checklist como uma barreira importante para a segurança no ambiente cirúrgico em 2018, contra 34,7\% (174) em 2012 (-Tabela 3); e 4. 55\% dos cirurgiões respondentes (402) referem fazer uso desta ferramenta com regularidade, contra 40,2\% (301) em 2014 (-Tabela 4).

\section{Discussão}

As pesquisas que envolvem populações específicas apresentam limitações. Nesta pesquisa, houve a participação de um percentual limitado, 18,7\% (730), da população-alvo, resultado superior ao obtido nas ações realizadas pela AA0-HNS, de $18,6 \%$, pela AAOS, de $16,6 \%{ }^{15}$ e pela SBOT em 2012 , de $15,5 \%(502) .{ }^{13} \mathrm{~A}$

Tabela 2 Profissionais que relataram desconhecer o Protocolo da Cirurgia Segura da Organização Mundial de Saúde

\begin{tabular}{|l|l|l|l|}
\hline & 2012 & 2014 & 2018 \\
\hline $\begin{array}{l}\mathrm{N}^{\circ} \text { total de } \\
\text { respondentes }\end{array}$ & 502 & 748 & 730 \\
\hline $\begin{array}{l}\mathrm{N}^{\circ} \text { dos que relataram } \\
\text { desconhecer } \\
\text { o protocolo }\end{array}$ & $\begin{array}{l}328 \\
(65,3 \%)\end{array}$ & $\begin{array}{l}341 \\
(45,6 \%)\end{array}$ & $\begin{array}{l}151 \\
(20,7 \%)\end{array}$ \\
\hline
\end{tabular}


Tabela 3 Profissionais que conhecem o protocolo da Organização Mundial de Saúde e o reconhecem como barreira de segurança

\begin{tabular}{|l|l|l|l|}
\hline & 2012 & 2014 & 2018 \\
\hline $\mathrm{N}^{\circ}$ total de respondentes & $\mathbf{5 0 2}$ & $\mathbf{7 4 8}$ & $\mathbf{7 3 0}$ \\
\hline $\begin{array}{l}\mathrm{N}^{\circ} \text { dos que conhecem o } \\
\text { protocolo e o reconhecem } \\
\text { como barreira de segurança }\end{array}$ & $\begin{array}{l}174 \\
(34,7 \%)\end{array}$ & $\begin{array}{l}407 \\
(54,4 \%)\end{array}$ & $\begin{array}{l}505 \\
(69,1 \%)\end{array}$ \\
\hline
\end{tabular}

Tabela 4 Profissionais que utilizam o protocolo da Organização Mundial de Saúde com frequêmcia

\begin{tabular}{|l|l|l|l|}
\hline & 2012 & 2014 & 2018 \\
\hline $\mathrm{N}^{\circ}$ total de respondentes & 502 & 748 & 730 \\
\hline $\begin{array}{l}\mathrm{N}^{\circ} \text { dos que utilizam o } \\
\text { protocolo com frequência }\end{array}$ & $\begin{array}{l}\text { Sem } \\
\text { dados }\end{array}$ & 301 & 402 \\
$(40,2 \%)$ & $(55,0 \%)$ \\
\hline
\end{tabular}

utilização dos padrões empregados por estas três sociedades teve por objetivo dar mais consistência às informações coletadas e permitir a comparação dos achados, principalmente entre as 3 pesquisas da SBOT, realizadas em 2012, 2014 e 2018.

Os participantes da pesquisa estão concentrados nos Estados de São Paulo, Rio de Janeiro e Minas Gerais (64,3\%), o que está de acordo com a distribuição geográfica dos ortopedistas no País. Da mesma forma, os especialistas com residência médica representam 78,4\% (572) do total dos respondentes correspondendo ao número de membros da SBOT que costuma comparecer ao congresso brasileiro. A quantidade de profissionais que informou já ter vivenciado, em algum momento de suas carreiras, cirurgias em local ou paciente errado representou 34,7\% (253) do total. Na pesquisa da AAOS, os erros referentes a cirurgias no lado errado representaram 59,1\% dos incidentes; esse percentual foi de $56 \%$ no estudo da Joint Commission on the Accreditation of Healthcare Organizations (Comissão Conjunta de Acreditação de Organizações de Saúde, JCAHO, em inglês), ${ }^{16}$ e de 40,8\% (205) no estudo de 2012 da SBOT. ${ }^{13}$

Relatos das sociedades americanas de subespecialidades também corroboram estes achados. A American Society for Surgery of the Hand (Sociedade Americana de Cirurgia da Mão, ASSH, em inglês) relatou 21\% de cirurgias em locais errados. ${ }^{17}$ Em cirurgia da coluna, tal número se mostra ainda mais alarmante, segundo pesquisa da American Academy of Neurologic Surgeons (Academia Americana de Cirurgiões Neurológicos), com 50\% dos respondentes informando já ter realizado pelo menos uma vez cirurgia em nível errado. ${ }^{18,19}$ A pesquisa realizada pela American Academy of Foot and Ankle Surgeons (Academia Americana de Cirurgiões de Pé e Tornozelo) também mostrou uma incidência de $13 \%$ de cirurgia em local errado. ${ }^{20}$

0 presente estudo permite concluir que membros das subespecialidades que apresentam maior frequência de cirurgias em locais errados, tais como a cirurgia da mão, da coluna vertebral e do pé e tornozelo, representaram um percentual menor de respondentes. Essa informação corrobora a ideia de que é preciso dedicar maior atenção a esses grupos de subespecialistas, pois as características anatômicas dessas regiões favorecem os erros, como corrobora a literatura. ${ }^{17-20}$

0 número de respondentes com menos de 5 anos de atuação, $28,9 \%$ do total, foi uma surpresa, uma vez que era esperado que profissionais mais jovens estivessem mais próximos da cultura da segurança, tema absolutamente atual.

No total, 423 dos ortopedistas que responderam (58\%) espontaneamente à pesquisa não estava envolvido com as atividades científicas do congresso. A premissa de que os profissionais que atuaram como palestrantes, teoricamente mais próximos dos círculos acadêmicos, estariam mais interessados em participar e transmitir seu conhecimento sobre o tema Segurança do Paciente, não se confirmou pelos percentuais encontrados, pois somente $42 \%$ (307) dos respondentes atuaram como palestrantes.

Ao todo, $624(85,5 \%)$ ortopedistas da amostra informaram marcar o local a ser operado antes de encaminhar o paciente ao centro cirúrgico, enquanto quase o mesmo percentual, 599 (82\%), relatou conferir o material de implante e o funcionamento dos equipamentos da sala antes da anestesia, o que demonstra uma certa maturidade na percepção do risco.

A categoria de erro mais frequente foi relacionada a material para uso cirúrgico incompleto ou danificado, identificado após o início do procedimento, seguida por problemas nos equipamentos ou instrumentos da sala cirúrgica. Nos achados da AAOS, em países desenvolvidos, erros relacionados a equipamentos são a falha mais comum, representando $29 \%$ do total, seguidos de erros de comunicação $(24,7 \%) .{ }^{15}$ Por outro lado, a categoria de erro mais frequente no meio ortopédico brasileiro, ou seja, material cirúrgico incompleto ou danificado, não é uma situação com representatividade nos Estados Unidos.

No total, $151(20,7 \%)$ ortopedistas afirmaram ainda desconhecer total ou parcialmente o protocolo da OMS, e 365 (50\%) mencionaram não ter tido treinamento para a sua utilização. Estes percentuais demonstram que ainda há a necessidade de capacitação de cirurgiões para a utilização desta ferramenta.

Os resultados encontrados neste estudo sugerem que a campanha educacional teve impacto positivo na melhoria da segurança no ambiente cirúrgico, mas que a SBOT ainda tem um longo caminho a percorrer, pois a utilização regular de barreiras contra erros consiste em uma mudança cultural, que só é possível por meio de ações de conscientização e treinamentos sistemáticos envolvendo não apenas os cirurgiões, como todos os profissionais ligados à atividade cirúrgica.

Um estudo ${ }^{21}$ similar, realizado no Reino Unido em 2010, já havia evidenciado que programas educacionais têm o potencial de aumentar significantemente não apenas o uso do checklist, como também a percepção positiva sobre sua utilização por membros da equipe.

Uma iniciativa de atualização dos cirurgiões ortopédicos acerca dos princípios e fundamentos de segurança na prática cirúrgica, que reconheceu oportunidades significativas para melhoria da qualidade, segurança e valor do cuidado oferecido às crianças, realizada pela Pediatric Orthopaedic Society of North America (Sociedade de Ortopedia Pediátrica da América do Norte, POSNA, em inglês) em 2016, sinaliza que a SBOT está no caminho correto em busca desta mudança cultural. ${ }^{22}$

Além disso, os resultados da revisão sistemática realizada pela POSNA em 2018, que selecionou, de acordo com o grau de contribuição para a melhoria da segurança e da qualidade do cuidado, 36 trabalhos científicos, é demonstração clara da importância destas iniciativas. ${ }^{23}$ 
Outra iniciativa impactante neste sentido seria a inclusão no currículo do tema qualidade e segurança no treinamento e na capacitação de residentes. ${ }^{24}$ Apesar de o compromisso e a excelência em nível individual serem essenciais, o ortopedista deve se preocupar com sistemas e protocolos que possam prover maior valor ao cuidado prestado.

Dentro deste contexto, a SBOT lançou, em 2018, um tratado intitulado Ortopedia e Traumatologia, que contém um capítulo inteiro dedicado à "Segurança do Paciente", ${ }^{25}$ o que, além de ressaltar a importância do tema, é de grande valia para a formação de novos ortopedistas.

\section{Conclusões}

Erros médicos ocorrem e representam risco para a segurança dos pacientes. A pesquisa aplicada demonstrou que é necessária uma mudança cultural no ambiente cirúrgico, para a melhoria da segurança não apenas dos pacientes, como também dos profissionais e das instituições.

O primeiro passo para esta mudança cultural é o reconhecimento dos erros e da possibilidade de aprender com eles. Neste sentido, as pesquisas sinalizam um grau de maturidade dos ortopedistas, pois, em todas as pesquisas, mais de $70 \%$ dos profissionais respondentes relata já ter vivenciado erros no ambiente cirúrgico.

Os dados do estudo mostram ainda que os esforços da SBOT para aumentar a percepção do risco envolvido no ambiente cirúrgico, preconizando a utilização do checklist cirúrgico da OMS como barreira de segurança, por meio da campanha “Considere o Risco," tiveram um impacto positivo, aumentando sensivelmente o número de ortopedistas que reconhecem o Protocolo de Cirurgia Segura como uma barreira de segurança e fazem uso frequente desta ferramenta.

Paralelamente, a pesquisa sinaliza ainda a necessidade de orientação relacionada ao tema, com treinamentos e capacitações sistemáticas, principalmente em se tratando dos jovens ortopedistas em formação, para que haja uma mudança cultural no ambiente cirúrgico.

Esta mudança, alcançável apenas por meio de ações sistemáticas direcionadas não apenas aos cirurgiões, como também a todos os profissionais envolvidos na atividade cirúrgica, é especialmente indicada e desejada em ortopedia e traumatologia, responsáveis por grande parte dos eventos adversos cirúrgicos, a maioria evitável por meio da utilização do Protocolo de Cirurgia Segura da OMS.

Conflito de interesses

Os autores declaram não haver conflito de interesses.

\section{Referências}

1 American Academy of Orthopaedic Surgeons. Information statement 1015. Wrong-site surgery. 2003 Out. Disponível em: http:// www.aaos.org/about/papers/advistmt/1015.asp [acesso em 26 de março de 2013]

2 Wong D, Herndon J, Canale T. An AOA critical issue. Medical errors in orthopaedics: practical pointers for prevention. J Bone Joint Surg Am 2002;84(11):2097-2100

3 Herndon JH. One more turn of the wrench. J Bone Joint Surg Am 2003t85(10):2036-2048
4 Kohn LT, Corrigan JM, Donaldson MS, Eds. To err is human: building a safer health system. Washington, DC: National Academy Press; 2000

5 World Health Organization. World Alliance for Patient Safety: Forward Programme, 2008-2009. Disponível em: www.who.int/ patientsafety/en/ [Acesso em 10 de dezembro de 2018]

6 ANVISA - Portal Segurança do Paciente. Disponível em: https:// www20.anvisa.gov.br/segurancadopaciente/index.php/noticias/ 60-cirurgiasseguras-salvam-vidas [Acesso em 02 de junho de 2019]

7 Gawande AA, Thomas EJ, Zinner MJ, Brennan TA. The incidence and nature of surgical adverse events in Colorado and Utah in 1992. Surgery 1999;126(01):66-75

8 Kable AK, Gibberd RW, Spigelman AD. Adverse events in surgical patients in Australia. Int J Qual Health Care 2002;14(04):269-276

9 Catchpole K, Mishra A, Handa A, McCulloch P. Teamwork and error in the operating room: analysis of skills and roles. Ann Surg 2008; 247(04):699-706

10 Lingard L, Espin S, Whyte S, et al. Communication failures in the operating room: an observational classification of recurrent types and effects. Qual Saf Health Care 2004;13(05):330-334

11 Panesar SS, Noble DJ, Mirza SB, et al. Can the surgical checklist reduce the risk of wrong site surgery in orthopaedics?-Can the checklist help? Supporting evidence from analysis of a national patient incident reporting system. J Orthop Surg Res 2011;6:18

12 Santiesteban L, Hutzler L, Bosco JA 3rd, Robb W 3rd. Wrong-Site Surgery in Orthopaedics: Prevalence, Risk Factors, and Strategies for Prevention. JBJS Rev 2016;4(01):pii: 01874474-201601000-00003

13 Motta Filho G, Silva LF, Ferracini AM, Bahr GL. Protocolo de Cirurgia Segura da OMS: o grau de conhecimento dos ortopedistas brasileiros. Rev Bras Ortop 2013;48(06):554-562

14 Shah RK, Kentala E, Healy GB, Roberson DW. Classification and consequences of errors in otolaryngology. Laryngoscope 2004; 114(08):1322-1335

15 Wong DA, Herndon JH, Canale ST, et al. Medical errors in orthopaedics. Results of an AAOS member survey. J Bone Joint Surg Am 2009;91(03):547-557

16 The Joint Commission. Universal Protocol. Disponível em: http:// www.jointcommission.org/standards_information/up.aspx [Acesso em 10 de dezembro de 2018]

17 Meinberg EG, Stern PJ. Incidence of wrong-site surgery among hand surgeons. J Bone Joint Surg Am 2003;85(02):193-197

18 Wong DA. Spinal surgery and patient safety: a systems approach. J Am Acad Orthop Surg 2006;14(04):226-232

19 Mody MG, Nourbakhsh A, Stahl DL, Gibbs M, Alfawareh M, Garges $\mathrm{KJ}$. The prevalence of wrong level surgery among spine surgeons. Spine 2008;33(02):194-198

20 Schweitzer KM Jr, Brimmo O, May R, Parekh SG. Incidence of wrong-site surgery among foot and ankle surgeons. Foot Ankle Spec 2011;4(01):10-13

21 Sewell M, Adebibe M, Jayakumar P, et al. Use of the WHO surgical safety checklist in trauma and orthopaedic patients. Int Orthop 2011;35(06):897-901

22 Glotzbecker MP, Wang K, Waters PM, McCarthy J, Flynn JM, Vitale MG; POSNA committee on the Quality, Safety, Value Initiative (QSVI). Quality, Safety, and Value in Pediatric Orthopaedic Surgery. J Pediatr Orthop 2016;36(06):549-557

23 Miller DJ, Cahill PJ, Janicki JA, Stephenson LP, Choi PD. What's New in Pediatric Orthopaedic Quality, Safety, and Value? A Systematic Review With Results of the 2016 POSNA Quality, Safety, and Value Initiative (QSVI) Challenge. J Pediatr Orthop 2018;38(10): e646-e651

24 Black KP, Armstrong AD, Hutzler L, Egol KA. Quality and Safety in Orthopaedics: Learning and Teaching at the Same Time: AOA Critical Issues. J Bone Joint Surg Am 2015;97(21):1809-1815

25 Bahr GL. Segurança na Prática Cirúrgica Ortopédica. In: Motta G, Barros T. Ortopedia e Traumatologia. Rio de Janeiro: Elsevier; 2018 